\title{
Characteristics of the Elastic Response of Isotropic Linear Micropolar Solids under Imposed Loads
}

\author{
Juan Alejandro Vazquez-Feijoo ${ }^{1}$, Pedro Montes-García ${ }^{1}$, Pator Teodoro Matadamas-Ortiz ${ }^{1}$, \\ Nicolas Juárez-Rodríguez ${ }^{2}$, Luicita Lagunez-Rivera ${ }^{1}$ \\ ${ }^{1}$ Centro Interdisciplinario de Investigación para el Desarrollo Integral Regional, CIIDIR-Oaxaca, \\ IPN, Engineering Group, Oaxaca, Mexico \\ ${ }^{2}$ Instituto Tecnológico de Tuxtla Gutiérrez, Tuxtla Gutiérrez, Mexico \\ Email: javazquezfeijoo@yahoo.xom.mx
}

Received April 12, 2013; revised May 29, 2013; accepted June 24, 2013

Copyright (c) 2013 Juan Alejandro Vazquez-Feijoo et al. This is an open access article distributed under the Creative Commons Attribution License, which permits unrestricted use, distribution, and reproduction in any medium, provided the original work is properly cited.

\begin{abstract}
This article proposes a simplified way to solve solid mechanic problems in micropolar elasticity using the solution found in the classic theory of elasticity as a starting point. In this study, an analysis of the linear isotropic micropolar elasticity is conducted based on the properties imposed on the micropolar medium by the constitutive and equilibrium equations. To ascertain how the micropolar medium responses deviate from Hooke's theory of elasticity, different loading conditions were classified. Three cases have been found so far: the rotational couple response, the quasi-classic equilibrium of momentum response and the general case. This study is the first in a series planned to explore the use of commercial packages of finite element in order to solve micropolar elasticity problems.
\end{abstract}

Keywords: Strength of Materials; Elasticity; Microcontinuum; Micropolar

\section{Introduction}

There are a number of elasticity theories which are based on the assumption that a solid is a continuous medium [1]. One such theory is the classic elasticity theory based on Hooke's law and is the one on which most of the commonly used materials in engineering are based. This theory assumes that each point on the continuum possesses three degrees of freedom or displacements in three orthogonal directions. Some special materials with no symmetry in their microstructure cannot be properly modelled using the classic theory, because they present additional mechanisms which oppose to deformation. That is the case in some cellular structures such as bones, wood and special materials like liquid crystal elastomers. In the case of human bones, several factors affect the mechanical response among which are age, gender and density. Because of these factors there is a wide range in the variation among the mechanical properties of this solid [2] and alternative elasticity theories should be used to model them properly. Microcontinuum theories are one such alternative.

These theories posit that each point of a continuous medium exhibits more than three degrees of freedom. On a prefatory note these theories consider that additional degrees of freedom have to be considered from the directors which are understood to be the orientations of the medium points. The microcontinuum theories can be classified as: 1) micropolar elasticity that assumes rigid medium points that can rotate; 2) microstretch elasticity that assumes breathing directors; and 3) micromorphic elasticity that assumes fully deformable directors [3].

The theory of micromorphic elasticity states that all the points in a solid have three displacements $u_{k}$. It also states that solids have nine other independent degreesof-freedom that are accounted in a $3 \times 3$ matrix containing the so-called director's components $x_{k K}$. Considering the simplest case in microcontinuum elasticity, the micropolar elasticity theory there are only three additional degrees of freedom namely the microrotations of the directors $\phi_{k}$.

To date, most of the microcontinuous studies have been based on the theory of micropolar elasticity, which has been applied to granular composites [4] or to cellular and porous materials [5]. Alternatively, the theories of microstretch and micromorphic elasticity have been applied to liquid crystal and liquid crystal elastomers [6,7]. The liquid crystal elastomers have attracted a great deal 
of attention, because of their remarkable damping properties [8].

There have been a number of studies done on micropolar elasticity [8-10]; however, most of them are focused on mathematical solutions to the equilibrium equations in particular cases. The work developed to date is sufficient for purposes of identification, but when the objective of the work is for purposes of prediction, particularly for engineering design, the studies may not be sufficient mainly because they are not clear about the conditions under which the assumptions are still valid. There are doubts about some of the static results obtained which assume that for a particular case, the displacements obtained from the classic elasticity theory are valid for micropolar linear isotropic solids. The assumption is that these displacements obey an equilibrium of momentum equation similar to those obtained from the theory of elasticity. It is necessary, however, to investigate under what conditions this assumption is valid. There are other assumptions which may not be valid as well, as in the case of pure bending, the macrorotation and microrotation are considered to be of the same magnitude (rotational coupled case).

This paper analyses the conditions which the micropolar constitutive and equilibrium equations impose on the theoretical micropolar solid and it is intended, from an engineering point of view, to enhance the predictability of the behaviour of the micropolar solid, so that a design can be feasible.

A micropolar solid is understood to be a solid in which the microstucture is regarded as rigid non-symmetric particles in the elastic medium. Only Cartesian coordinates are used here. In the first section, the equations that conform to the linear isotropic micropolar elastic theory are presented. In the second section, the conditions under which a micropolar solid behaves as a case of rotational coupled are considered. In the third section, the focus is on the determination of the condition that does not conform to the rotational coupled behaviour. This latter is still a classical-type equilibrium of momentum equation and, therefore, a displacement field similar to the classical elasticity, can be assumed. The final section presents a methodology to solve iteratively all other loading conditions that do not fall under the cases considered in the previous sections; however it is still an exact solution.

\section{Micropolar Elasticity Theory Review}

This section concentrates on the Linear Isotropic Micropolar Elasticity Theory and presents the equations that are used in the remaining part of the paper. Fundamentals of this theory can be found in [3]. Micropolar theory is based on two constitutive equations, two equilibrium equations and two compatibility equations. Each of the medium points possesses six degrees-of-freedom: three displacements $u_{x}, u_{y}, u_{z}$ and three microrotations $\phi_{x}, \phi_{y}$ and $\phi_{z}$.

The constitutive equations are,

$$
\begin{aligned}
& t_{k l}=\lambda \varepsilon_{r r} \delta_{k l}+(\mu+\kappa) \varepsilon_{k l}+\mu \varepsilon_{l k} \\
& m_{k l}=\alpha \phi_{r, r} \delta_{k l}+\beta \phi_{k, l}+\gamma \phi_{l, k}
\end{aligned}
$$

in which the comma indicates the usual partial derivative and the repeated sub-indices imply a summation. These equations define six constitutive parameters $\lambda, \mu, \kappa \alpha, \beta$ and $\gamma$ that are needed to guarantee positive internal energy. This restriction imposes the following conditions,

$$
\begin{aligned}
& 0 \leq 3 \lambda+2 \mu+\kappa \quad 0 \leq 2 \mu+\kappa \quad 0 \leq \kappa \\
& 0 \leq 3 \alpha+\beta+\gamma \\
& -\gamma \leq \beta \leq \gamma \quad 0 \leq \gamma
\end{aligned}
$$

The microstrain tensor $\varepsilon_{k l}$ that appears in Equations (1) and (2) is defined as,

$$
\varepsilon_{k l}=-\epsilon_{k \ln } \phi_{n}+u_{l, k}
$$

As can be observed from Equation (4), the microstrain tensor possesses an antisymmetric component due to the microrotations of the medium points.

The local equilibrium equations are:

The equilibrium of momentum,

$$
\begin{aligned}
& t_{l k, l}+\rho\left(f_{k}-\ddot{u}_{k}\right)=0 \quad \text { (in V) } \\
& t_{l k} n_{l}=t_{(n) k} \quad \text { (in S) }
\end{aligned}
$$

and the equilibrium of the moment of momentum,

$$
\begin{aligned}
& m_{l k, l}+\epsilon_{k m n} t_{m n}+\rho\left(l_{k}-j \ddot{\phi}_{k}\right)=0 \quad(\text { in } \mathrm{V}) \\
& \left.m_{l k} n_{l}=m_{(n) k} \quad \text { (in S }\right)
\end{aligned}
$$

this case, $\mathrm{V}$ is the volume of the micropolar medium and $\mathrm{S}$ is the boundary of the micropolar medium.

Microrotation and displacements can also be prescribed. In Equation (6) $l_{k}$ represents induced microcouples per unity of mass and $j$ is the microinertia, which is a constant for the linear theory. The compatibility equations are,

$$
\varepsilon_{i k, j}-\varepsilon_{j k . i}+\epsilon_{i k n} \phi_{n, j}-\epsilon_{j k n} \phi_{n, i}=0
$$

and,

$$
\epsilon_{k l r}\left(\in_{k \ln } \phi_{n, m, i}-\epsilon_{k l i} \phi_{n, i, m}\right)=0
$$

\section{Rotational Coupled Case in a Micropolar Elastic Solid}

Assuming that the displacement field in the medium can be expressed by polynomial functions, the macrorotations $\left(R_{j}\right)$ depend on the displacement field described by the following equation: 


$$
R_{j}=\frac{1}{2} \in_{j k l} u_{l, k}
$$

and therefore the macrorotations can also be expressed through polynomial functions. Using this equation in Equation (4), the microstrain tensor can be expressed as a function of the rotations,

$$
\varepsilon_{k l}=e_{k l}+\epsilon_{k \ln }\left(R_{n}-\phi_{n}\right)
$$

For simplicity, consider that there are no microcouples $l_{j}$. Substituting Equations (1) and (2) into (5) and (6), respectively, and using Equation (9) the equation of moment of momentum for the static case, can be rewritten as,

$$
R_{j}=\phi_{j}-\frac{1}{2 \kappa}\left[(\alpha+\beta) \phi_{k, k j}+\gamma \phi_{j, k k}\right]
$$

In this section the rotational coupled case is revised. The revision considers the convenient condition, from a mathematical point of view, that the macrorotations and microrotations are of the same magnitude, i.e.,

$$
R_{j}=\phi_{j}
$$

Once this has been established it is possible to deduce that the conditions under Equation (12) are true. According to the Equation (11), the macrorotations are a function of the microrotations and their second order derivatives, as for example $\phi_{k, k j}$. If according to the boundary conditions, the displacement field can be expressed as a polynomial function of less than a second degree of the coordinates, all the second order derivatives are zero; therefore, Equation (11) reduces to Equation (12). Several static cases already reported indicate that simple tension, torsion and bending [11] fell into this case, and only the Equation (5) is needed to solve the displacement field.

As a consequence of Equation (12), Equation (10) is now, simply,

$$
\varepsilon_{k l}=e_{k l}
$$

The symmetric strain tensor $e_{k l}$ is obtained from the Green stretch tensor. The antisymmetric part of Equation (10) disappears.

An extreme example of the rotational coupled case is when a micropolar beam is under pure bending [10], which undertakes a macrorotation given by,

$$
R_{j}=a x_{k}
$$

Substitution of Equation (14) in Equation (11) gives,

$$
\phi_{j}=a x_{k}
$$

It should be clarified that there is no intention, at this point, to give a particular example of particular conditions. Rather, the intention is to consider the general results obtained from the constitutive equations. The ex- amples given through references are provided only to demonstrate that there are particular cases that fall into the polynomial case considered in this work.

The antisymmetric part of the microstrain from Equation (10) is not considered; therefore, Equation (5) reads,

$$
\lambda e_{k k, j}+(2 \mu+\kappa) e_{k j, k}=0
$$

Considering,

$$
\mu_{c}=\mu+\frac{\kappa}{2}
$$

Equation (17) is similar to the classical elasticity equilibrium equation,

$$
\lambda e_{k k, j}+2 \mu_{c} e_{k j, k}=0
$$

and therefore it can be assumed that there are identical fields of deformation for both classic elasticity and micropolar (for the case of the rotational coupled) theories. As the anti-symmetric part of the strains is zero (equation 12 ), the macrorotation does not contribute to the resistance of stresses produced by external forces (Equation (1)). The microrotations on the other hand do produce resistance against external moments or microcouples applied to the body (Equation (2)).

It can be concluded that a necessary condition of a micropolar solid, to respond as a rotational coupled case, is that the macro-rotation has to be a first-degree polynomial function of one of the coordinates $x_{j}$ or a constant. This implies that the static cases of simple tension (macro-rotation is zero) and pure beam bending (a first order function) all fall into this category.

\section{Quasi-Classic Equilibrium of Momentum}

The rotational coupled case has an advantageous circumstance because in this situation the same deformation assumptions used in the classical elasticity analysis can also be assumed in the analogue cases for the micropolar elasticity. This is possible because the equation of equilibrium of momentum resembles that of the classical elasticity theory; however, this is not the unique case in which it may happen.

Equation (5) can be expressed as function of the symmetric and antisymmetric strain tensors as follows,

$$
\begin{aligned}
& \lambda e_{k k, j}+(2 \mu+\kappa) e_{k j, k}+\kappa \in_{k j l}\left(R_{l, k}-\phi_{l, k}\right) \\
& +\kappa \in_{l j k}\left(R_{k, l}-\phi_{k, l}\right)=0
\end{aligned}
$$

For clarity, concentrated forces are eliminated in Equation (19). Note that the classical equilibrium equations appears once more if,

$$
R_{l, k}-\phi_{l, k}=R_{k, l}-\phi_{k, l}
$$

By the use of Equation (11), Equation (21) can be obtained, 


$$
\begin{aligned}
& (\alpha+\beta) \phi_{j, j l k}+\gamma \phi_{l, j j k} \\
& =(\alpha+\beta) \phi_{j, j k l}+\gamma \phi_{k, j j l}
\end{aligned}
$$

which can be simplified as,

$$
\phi_{l, j j k}=\phi_{k, j j l}
$$

This equation holds for any field of displacements that produces a macrorotation with a polynomial function of a third degree or lower because third and higher order derivatives become zero. An example of this is a Timoshenko beam with two simple supports bearing concentrated force. The displacement field in the classical elasticity theory (Hooke's Law) consists of polynomials of a maximum degree of three. A similar field can be assumed for the micropolar medium. With the Timoshenko beam the flexural moment is considered to be a function of the first order of the axe 1 which coincides with the largest dimension of the beam along the neutral axe. It can be written,

$$
M_{3}(x)=V_{0} x_{1}+M_{0}
$$
tion,

The stress can be obtained from the well known equa-

$$
\sigma_{11}=\frac{M\left(x_{1}\right)}{I_{3}} x_{2}
$$

Here the axe 2 is oriented in the direction of the loads. The displacement in the $x_{1}$ direction is,

$$
u_{1}=\frac{1}{E I_{3}}\left(\frac{V_{0}}{2} x_{1}^{2} x_{2}+M_{0} x_{2}\right)
$$

which implies a macrorotation of,

$$
R_{3}=\frac{1}{E I_{3}}\left(\frac{V_{0}}{2} x_{1}^{2}+M_{0}\right)
$$

which gives a macrorotation expressed by a second degree polinomial function. In a general approach, if the macrorotation can be expressed as a second degree polynomial,

$$
R_{j}=a x_{i}^{2}
$$

the moment of momentum Equation (11) is,

$$
a x_{i}^{2}=a x_{i}^{2}+C-\frac{1}{2 \kappa}\left[(\alpha+\beta) \phi_{k, k j}+\gamma \phi_{j, k k}\right]
$$

where, the microrotation is assumed to be,

$$
\phi_{j}=a x_{i}^{2}+C
$$

Because there is no other possible second degree term in Equation (25). Here, $C$ is a constant that must balance the second term of the left hand side of Equation (25),

$$
C=2 \gamma a
$$

therefore,

$$
\phi_{j}=a x_{i}^{2}+2 \gamma a
$$

The substitution of Equation (26) in Equation (19) gives,

$$
\begin{aligned}
& \lambda e_{k k, j}+(2 \mu+\kappa) e_{k j, k} \\
& +\frac{1}{2}\left[\epsilon_{i j l}(0-0)+\kappa \epsilon_{i j k}\left(2 a x_{i}-2 a x_{i}\right)\right]=0
\end{aligned}
$$

then, by the use of Equation (17),

$$
\lambda e_{k k, j}+\mu_{c} e_{k j, k}=0
$$

which is analogous to the classical elasticity equation of equilibrium. Therefore, the same assumptions can be considered for the micropolar medium.

Because of the restriction on space, more details on this beam are not included in this paper; however, if further information is needed it can be found elsewhere.

If the microrotation is a function of time $\phi_{l, k}(t)$, as in the case of wave propagation, the microinertia has to be considered (Equation (6)). The condition for holding Equation (20) is restricted to,

$$
\phi_{l, k}=-\phi_{k, l}
$$

Another case where Equation (27) can also be applied is when there is a constant microcouple per unit of volume, as observed by analysing Equation (11). A constant microcouple does not produce strains. No excitation of the micropolar body appears either in the equilibrium equation of momentum (Equation (27)) or in the boundary conditions (5b) and (6b). The microcouple is equilibrated only by the microrotation

Analogous to the classic elasticity theory, Eringen [9] considers the case of micropolar elasticity for plane strain, in which the displacements possess the following restrictions,

$$
\begin{aligned}
& \phi_{\alpha}=\phi_{\beta}=0 \quad \phi_{z}=f\left(x_{1}, x_{2}\right) \\
& \varepsilon_{\alpha z}=\varepsilon_{z \alpha=0}
\end{aligned}
$$

The condition stated by Equation (22) is reduced to,

$$
\phi_{z, \alpha \alpha \beta}=0
$$

This implies that both sides of (21) are zero and, if so represents the rotational coupled case.

If the quasi-classic equilibrium equation is valid, then the deformations are analogous to those obtained by the classical elasticity theory; however, when the macro and microrotations are not coupled, then the microrotations are opposed to the external loads by producing microcouples and/or shear stress, as the antisymmetric part of the strains is no longer zero, see Equation (4).

\section{The General Linear Isotropic Micropolar Elastic Solid in Static Conditions}

The advantage of the cases treated in the previous sec- 
tions is that the proposed solution for a particular case can be extrapolated directly from the deformation field, which itself can be obtained from the classical elasticity theory. In the general case, the following considerations can be taken to solve the problems which are not considered in the previous cases. Referring back to Equation (11) and bringing back into this equation the induced microcouples per unit of mass, let's split both the macrorotation and microrotation into two terms. Then Equation (11) is now,

$$
\begin{aligned}
& R_{j}^{\prime}+R_{j}^{\prime \prime \prime}=\phi_{j}^{\prime}+\phi_{j}^{\prime \prime} \\
& -\frac{1}{2 \kappa}\left[(\alpha+\beta) \phi_{k, k j}+\gamma\right] \phi_{j, k k}-\frac{\rho}{2 \kappa} I_{j}
\end{aligned}
$$

Because this is a linear differential equation, by the use of the superposition principle, Equation (31) can be split into two equations as follows,

$$
R_{j}^{\prime}-\phi_{j}^{\prime}=-\frac{\rho}{2 \kappa} l_{j}
$$

and,

$$
R_{j}^{\prime \prime \prime}-\phi_{j}^{\prime \prime}=-\frac{1}{2 \kappa}\left[(\alpha+\beta) \phi_{k, k j}^{\prime}+\gamma \phi_{j, k k}^{\prime}\right]
$$

Considering Equation (32), the equilibrium of momentum given by Equation (19) is now,

$$
\lambda e_{k k, j}+(2 \mu+\kappa) e_{k j, k}=\epsilon_{k j l} \frac{\rho}{2} l_{j, k}+\epsilon_{l j k} \frac{\rho}{2} l_{k, j}
$$

A displacement vector $u_{j}^{\prime}$ is obtained. Observe that if there are any microcouples per unit of mass, this equation becomes the quasi-static equilibrium (Equation (18)) because the microcouples participate as field forces just as gravity does. From Equation (11), the macrorotation $R_{j}^{\prime}$ can be obtained. Then, $\phi_{j}^{\prime}$ is obtained with Equation (32).

Thereafter, if the microrotations $\phi_{j}^{\prime}$ possess a nonzero second order derivative, the Equation (33) can be substituted in Equation (19) thus obtaining,

$$
\begin{aligned}
& \lambda e_{k k, j}+(2 \mu+\kappa) e_{k j, k}+\epsilon_{l j k}\left[(\alpha+\beta) \phi_{k, k j l}+\gamma \phi_{j, k k l}\right] \\
& +\epsilon_{k l j}\left[(\alpha+\beta) \phi_{j, j k l}+\gamma \phi_{k, j j l}\right]
\end{aligned}
$$

Equation (33) produces other sets of displacements $u_{j}^{\prime \prime}$ from which it is possible to calculate the microrotations $R_{j}^{\prime \prime \prime}$. The term $\phi_{j}^{\prime \prime}$ is now obtained using Equation (31).

If $\phi_{j}^{\prime \prime}$ has a non-zero second derivative in respect to the other two coordinates, then the process is repeated to obtain a new set of rotations, e.g. $R_{j}^{\prime \prime \prime}$ and $\phi_{j}^{\prime \prime}$, up to the point in which the corresponding second order derivative of the microrotation term is zero. The total displacement macrorotation and microrotation are,
a) $R_{j}=R_{j}^{\prime}+R_{j}^{\prime \prime}+R_{j}^{\prime \prime \prime}+\cdots$
b) $\phi_{j}=\phi_{j}^{\prime}+\phi_{j}^{\prime \prime}+\phi_{j}^{\prime \prime \prime}+\cdots$

and,

$$
u_{j}=u_{j}^{\prime}+u_{j}^{\prime \prime}+u_{j}^{\prime \prime \prime}+\cdots
$$

Now that the proposed methodology has been illustrated the discussion of some examples on the general linear isotropic micropolar elastic solids are presented.

\section{Examples or the General Linear Isotropic Micropolar Elastic Solids}

\subsection{Clamped Beam with a Free End and a Microcouple per Unit of Volume}

Suppose that a micropolar solid of dimensions $u_{0}, v_{0}$ and $w_{0},\left(w_{0} \gg u_{0}, v_{0}\right)$ is clamped at the end at $x=0$ and that there is a free end at $x=u_{0}$, as shown in Figure 1. Then, because of the magnetic or electric field, a non uniform microcouple per unit of volume is induced in accordance with,

$$
l_{z}=A x^{3}
$$

No other external forces are applied different from the reaction at $x=0$. By the use of Equation (34) one may start considering,

$$
\begin{gathered}
R_{z}^{\prime}=\phi_{z}^{\prime}-\frac{\rho}{2 \kappa} l_{z} \\
R_{z}^{\prime}-\phi_{z}^{\prime}=-\frac{\rho}{2 \kappa} A x^{3}
\end{gathered}
$$

Under this first approximation, Equation (19) is now,

$$
\begin{aligned}
& \text { a) } \lambda e_{k, k x}+(2 \mu+\kappa) e_{k, x k}=0 \\
& \text { b) } \lambda e_{k k, y}+(2 \mu+\kappa) e_{k y, k}=-\frac{3 \rho}{2} A x^{2} \\
& \text { c) } \lambda e_{k, k z}+(2 \mu+\kappa) e_{k, z k}=0
\end{aligned}
$$

The unique equation with non trivial solution is (38b). As the microcouple is only a function of $x$, it is assummed that all the displacements are a function only of $x$. This implies that a non-normal stress is present in this equation and it can be reduced to,

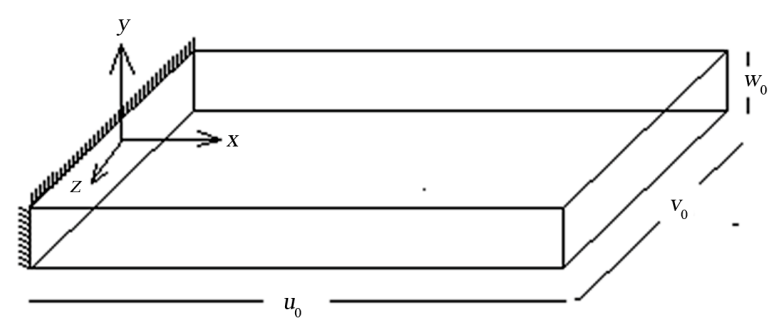

Figure 1. The micropolar solid is prevented from rigid displacement and then a distributed couple per unit of mass is applied. 


$$
(2 \mu+\kappa) e_{x y, x}=-\frac{3 \rho}{2} A x^{2}
$$

Considering that any displacement depends only on the direction $x$, the previous equation is reduced to,

$$
(2 \mu+\kappa) \frac{u_{y, x, x}}{2}=-\frac{3 \rho}{2} A x^{2}
$$

The unique nonzero displacement depends on $x$,

$$
u_{y, x x}=\frac{3 \rho A}{(2 \mu+\kappa)} x^{2}
$$

The displacement field in this first iteration is,

$$
u=\left\{0, \frac{\rho A}{4(2 \mu+\kappa)} x^{4}, 0\right\}
$$

The macrorotation $R_{z}^{\prime}$ is now,

$$
R_{z}^{\prime}=\frac{\rho A}{2(2 \mu+\kappa)} x^{3}
$$

Using Equation (32)

$$
\phi_{z}^{\prime}=\frac{\mu+\kappa}{\kappa(2 \mu+\kappa)} \rho A x^{3}
$$

Equation (33) then gives,

$$
R_{j}^{\prime \prime}-\phi_{j}^{\prime \prime}=-\frac{3 \gamma(\mu+\kappa)}{\kappa^{2}(2 \mu+\kappa)} \rho A x
$$

Equation (19) is now,

$$
\begin{aligned}
& \text { a) } \lambda e_{k, k x}+(2 \mu+\kappa) e_{k, x k}=0 \\
& \text { b) } \lambda e_{k k, y}+(2 \mu+\kappa) e_{k y, k}=\frac{3 \gamma(\mu+\kappa)}{\kappa(2 \mu+\kappa)} \rho A \\
& \text { c) } \lambda e_{k, k z}+(2 \mu+\kappa) e_{k, z k}=0
\end{aligned}
$$

The displacement is now,

$$
u^{\prime \prime}=\left\{0, \frac{3 \gamma(\mu+\kappa)}{2 \kappa(2 \mu+\kappa)^{2}} \rho A x^{2}, 0\right\}
$$

The macrorotation $R^{\prime \prime}{ }_{z}$ is,

$$
R_{z}^{\prime \prime}=\frac{3 \gamma(\mu+\kappa)}{2 \kappa(2 \mu+\kappa)^{2}} \rho A x
$$

The total macrorotation $R_{z}$ is (adding Equations (41) and (46)),

$$
R_{z}=\frac{\rho A}{2(2 \mu+\kappa)} x^{3}+\frac{3 \gamma(\mu+\kappa)}{2 \kappa(2 \mu+\kappa)^{2}} \rho A x
$$

Equations (40) and (45) give the displacement,

$$
u_{y}=\frac{\rho A}{4(2 \mu+\kappa)} x^{4}+\frac{3 \gamma(2 \mu+3 \kappa)}{4 \kappa(2 \mu+\kappa)^{2}} \rho A x^{2}
$$

Equations (43) and (47) give,

$$
\phi_{z}^{\prime \prime}=\frac{3 \gamma(4 \mu+5 \kappa)(\mu+\kappa)}{2 \kappa^{2}(2 \mu+\kappa)^{2}} \rho A x
$$

Adding Equations (42) and (49),

$\phi_{z}=\frac{\mu+\kappa}{\kappa(2 \mu+\kappa)} \rho A x^{3}+3 \gamma \frac{(4 \mu+5 \kappa)(\mu+\kappa)}{2 \kappa^{2}(2 \mu+\kappa)} \rho A x$

As derivatives in Equation (32) become zero after the third iteration, no further iterations are needed. In this analysis $e_{k k}=0$, so there is no normal stress as there is when external flexural moments are applied (Figure 2). As the displacement $u_{y}$ is a function only of $x$, only shear stresses are present and each produces a sliding cross section (Figures 3 and 4). Observe that this microcouple per unit of volume does not produce any reaction force in the clamped end, as no displacement is produced at $x=0$. This can be seen using Equation (4) together with Equation (1), from which is obtained,

$$
\begin{aligned}
t_{y x}= & \mu\left(\frac{1}{(2 \mu+\kappa)} x^{3}+\frac{\mu+\kappa}{(2 \mu+\kappa)}\right) \rho A x^{3} \\
& +\left(\frac{3 \gamma(2 \mu+3 \kappa)}{2 \kappa(2 \mu+\kappa)^{2}}+3 \gamma \frac{(4 \mu+5 \kappa)(\mu+\kappa)}{2 \kappa(2 \mu+\kappa)}\right) \rho A x
\end{aligned}
$$

However there is a moment, $M_{z}$, because of the presence of the microcorotation $\phi_{z x}$. The use of Equation (50) gives,

$$
\phi_{z, x}=3 \frac{\mu+\kappa}{\kappa(2 \mu+\kappa)} \rho A x^{2}+3 \gamma \frac{(4 \mu+5 \kappa)(\mu+\kappa)}{2 \kappa^{2}(2 \mu+\kappa)} \rho A
$$

The micromoment $m_{z x}$ is obtained from Equation (2),

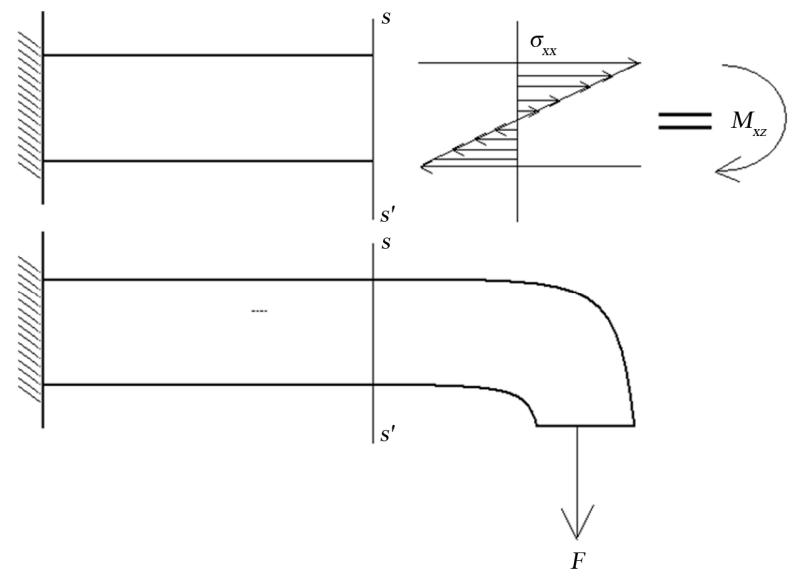

Figure 2. The moment $M_{x z}$ is applied through the distribution of $\sigma_{x x}$ on the boundary $s-s^{\prime}$. 


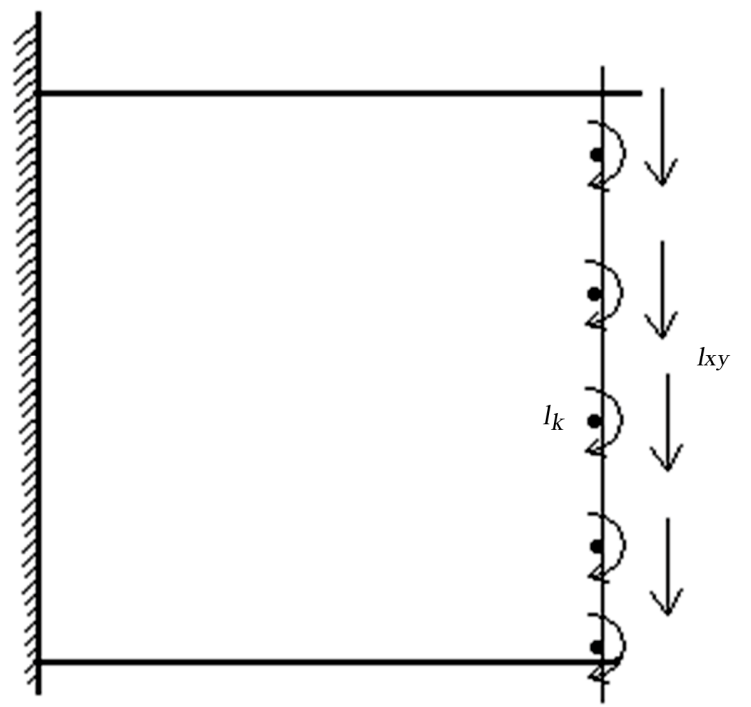

Figure 3. Concentrated microcouple per unit of volume produce only shear strain.

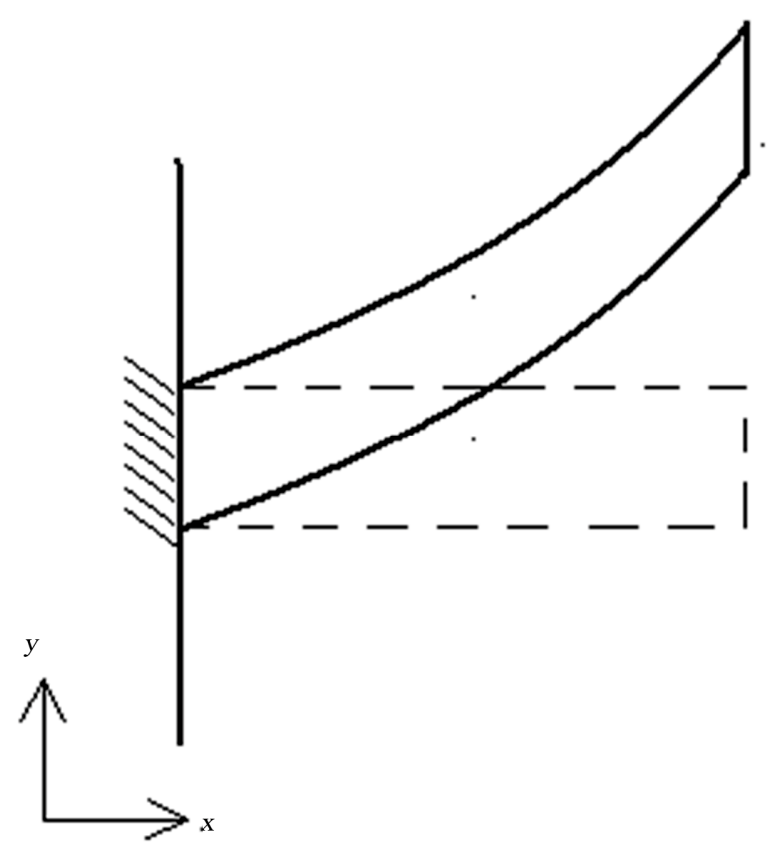

Figure 4. Deformation of the micropolar body under a cubic distributed couple per unit of volume.

$$
m_{z x}=3 \beta\left(\frac{\mu+\kappa}{\kappa(2 \mu+\kappa)} \rho A x^{2}+\gamma \frac{(4 \mu+5 \kappa)(\mu+\kappa)}{2 \kappa^{2}(2 \mu+\kappa)} \rho A\right)
$$

that when evaluated in $x=0$, the following is obtained,

$$
\left.m_{z x}\right|_{x=0}=3 \beta \gamma \rho A \frac{(4 \mu+5 \kappa)(\mu+\kappa)}{2 \kappa^{2}(2 \mu+\kappa)}
$$

From the previous equation the total moment $M_{z}$ can be obtained by integrating,

$$
M_{z}=\int_{z=0}^{z=w_{0}} 3 \beta \gamma \rho A \frac{(4 \mu+5 \kappa)(\mu+\kappa)}{2 \kappa^{2}(2 \mu+\kappa)} \partial z
$$

finally obtaining,

$$
M_{z}=3 \beta \gamma \rho A \frac{(4 \mu+5 \kappa)(\mu+\kappa)}{2 \kappa^{2}(2 \mu+\kappa)} w_{0}
$$

\subsection{Double Clamped Beam with a Concentrated Force}

The case of a doubled clamped beam (see Figure 5) is analyzed using the same assumptions considered in [10]. As noted before, the starting point of the analysis is the traditional elasticity theory, then, if the Euler-Bernoulli equation is used in the analysis, the shear force, the moment, the angular deflexion and the vertical displacement are as follows,
a) $V_{0 \rightarrow a}=V_{1}$
b) $V_{a \rightarrow b}=V_{1}-F$
c) $M_{0 \rightarrow a}=V_{1} x-M_{1}$
d) $M_{a \rightarrow b}=V_{1} x-M_{1}-F(x-a)$
e) $E_{m} I \theta_{0 \rightarrow a}=\frac{V_{1} x^{2}}{2}-M_{1} x$
f) $E_{m} I \theta_{a \rightarrow b}=\frac{V_{1} x^{2}}{2}-M_{1} x-\frac{F(x-a)^{2}}{2}$
g) $E_{m} I V_{0 \rightarrow a}=\frac{V_{1} x^{3}}{6}-\frac{M_{1} x_{2}}{2}$
h) $E_{m} I V_{a \rightarrow b}=\frac{V_{1} x^{3}}{6}-\frac{M_{1} x^{2}}{2}-\frac{F(x-a)^{3}}{6}$

where $E_{m}$ is the equivalent to the Young modulus,

$$
E_{m}=\frac{t_{k k}}{\varepsilon_{k k}}=\frac{(2 \mu+\kappa)(3 \lambda+2 \mu+\kappa)}{(2 \lambda+2 \mu+\kappa)}
$$

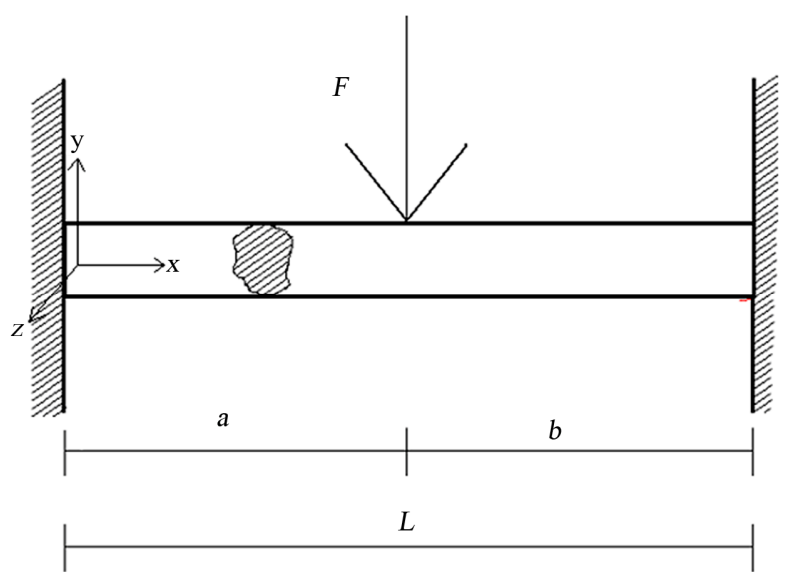

Figure 5. Double clamped beam with symmetric transverse area. 
This equation, similar to that of the classic elasticity theory, can be obtained from the matrix Equation (1) when inverted for deformations, see references $[8,9]$.

Given a specific cut around any cross section in $x$, there is a moment $M(x)$ that has to be equilibrated by the moment produced by the normal forces around the $z$-axis and the moment produced by the microcouples $m_{x z}$ distributed all around the face $x$. Therefore,

$$
M(x)=\int_{A_{x}} \sigma(x, y) y \mathrm{~d} A_{x}+\int_{A_{x}} m_{x z}(x, y) \mathrm{d} A_{x}
$$

As in the Euler Bernoulli beam, the normal stress is a linear function of $y$,

$$
\begin{gathered}
\sigma_{x x}=\sigma_{0}(x) y \\
M(x)=\int_{A_{x}} \sigma_{0}(x) y^{2} \mathrm{~d} A_{x}+\int_{A_{x}} m_{x z}(x, y) \mathrm{d} A_{x}
\end{gathered}
$$

In order to consider the application of Equation (2), and assuming that $R_{z}^{\prime}=\phi_{z}^{\prime}$, which is valid as a first approach,

$$
m_{x z}(x, y)=\gamma \phi_{z, x}^{\prime}(x, y)=\gamma R_{z, x}^{\prime}(x, y)
$$

The integration of this equation gives,

$$
M(x)=\sigma_{0}(x) I_{z}+\int_{A_{x}} \gamma R_{z, x}^{\prime}(x, y) \mathrm{d} A_{x}
$$

The macrorotation is given by,

$$
R_{z}^{\prime}(x, y)=\frac{1}{2}\left(\frac{\partial v(x, y)}{\partial x}-\frac{\partial u(x, y)}{\partial y}\right)
$$

because the unique displacement is in the $x$ direction $u(x, y)$, then $R_{z}^{\prime}(x, y)=-\frac{1}{2}\left(\frac{\partial u(x, y)}{\partial y}\right)$

and the integrals end to be,

$$
M(x)=\sigma_{0}(x) I_{z}-\frac{\gamma}{2} \int_{A_{x}} \frac{\partial^{2} u(y, x)}{\partial y \partial x} \mathrm{~d} A_{x}
$$

Considered in the classic treatment of bending (transverse areas $A_{x}$ remains without deformation). The displacement $u(y, x)$ can be obtained from the bending angle $\theta(x)$ as shown in Figure 6.

Taking into account the geometrical parameters shown in Figure 1, it can be established that

$$
\partial \theta=\frac{\partial x}{\rho}=-\frac{\partial u(x, y)}{y}
$$

Therefore,

$$
\frac{\partial u(x, y)}{\partial x}=-\frac{y}{\rho}
$$

The displacement $u(x, y)$ is then,

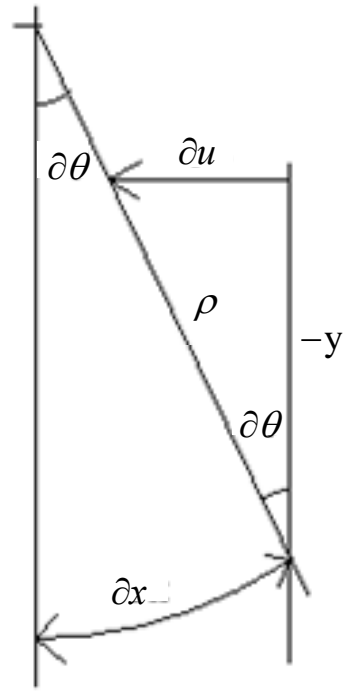

Figure 6. The displacement $u$ is function of the bending angle.

$$
u(x, y)=-y \int_{0}^{x} \frac{\partial \eta}{\rho(\eta)}
$$

The stress is then obtained as,

$$
\sigma_{x x}=E_{m} \varepsilon_{x x}
$$

Using Equation (56), the stresses are then obtained as,

$$
\sigma_{x x}=E_{m} \frac{\partial u(x, y)}{\partial x}=-\frac{y}{\rho(x)} E_{m}
$$

From Equation (52),

$$
\sigma_{0}(x)=-\frac{E_{m}}{\rho(x)}
$$

and with the use of Equations (57) and (60), Equation (54) is now,

$$
M(x)=-\left(1+\frac{\gamma}{2 E_{m} k_{z}^{2}}\right) \frac{E_{m} I_{z}}{\rho(x)}
$$

where $k_{z}$ is the well known radii of gyration.

The radii of curvature is,

$$
\frac{M(x)}{\varphi E_{m} I_{z}}=-\frac{1}{\rho(x)}
$$

where $\varphi$ is,

$$
\varphi=\left(1+\frac{\gamma}{2 E_{m} k_{z}^{2}}\right)
$$

Using Equations (52) and (60),

$$
\sigma(x, y)=-\frac{M(x)}{\varphi I_{z}} y
$$


and the integration of Equation (61) gives,

$$
\frac{1}{\varphi E_{m} I_{z}} \int_{0}^{x} M(\eta) \partial \eta=-\int_{0}^{x} \frac{\partial \eta}{\rho(\eta)}
$$

From Equation (55),

$$
\theta(x)=\int_{0}^{x} \partial \theta(\eta) \partial \eta=-\int_{0}^{x} \frac{\partial \eta}{\rho(\eta)}
$$

The substitution of (65) in (64) gives,

$$
\varphi E_{m} I_{z} \theta(x)=\int_{0}^{x} M(\eta) \partial \eta
$$

The displacement $u(x, y)$ is then in accordance with Equation (57),

$$
u(x, y)=-y \int_{0}^{x} \frac{\partial \eta}{\rho(\eta)}=y \theta(x)
$$

The macrorotation can be rewritten as,

$$
R_{z}^{\prime}(x)=\frac{1}{2}\left(\frac{\partial u(x, y)}{\partial y}\right)=\frac{1}{2} \theta(x)
$$

Now, equation (19) is valid, when $R_{z}^{\prime}(x, y)=\phi_{z}^{\prime}(x, y)$; therefore,

$$
\phi_{z}^{\prime}(x)=\frac{1}{2} \theta(x)
$$

Substituting Equation (67) in Equation (11),

$$
R_{z}^{\prime \prime}(x)=\phi_{z}^{\prime \prime}(x)-\frac{1}{2 \kappa}\left(\left[\gamma \phi_{z, x x}^{\prime}\right]\right)
$$

Considering Equation (67),

$$
\phi_{z, x x}^{\prime}(x)=\frac{1}{2} \frac{\partial^{2} \theta(x)}{\partial x^{2}}
$$

Using the Equation (66) and the basic concepts of mechanics of materials,

$$
\phi_{z, x x}^{\prime}(x)=\frac{1}{2 \varphi E_{m} I_{z}} \frac{\partial M(x)}{\partial x}=\frac{V(x)}{2 \varphi E_{m} I_{z}}
$$

Equation (68) can then be written as,

$$
R_{z}^{\prime}(x)=\phi_{z}^{\prime}(x)-\frac{\gamma V(x)}{4 \varphi \kappa E_{m} I_{z}}
$$

or,

$$
\phi_{z}^{\prime \prime}(x)=R_{z}^{\prime}(x)-\phi^{\prime}(x)=-\frac{\gamma V(x)}{4 \varphi \kappa E_{m} I_{z}}
$$

For the particular case of concentrated forces, as in the present example, all the derivatives of $V(x)$ are zero; therefore there is no possibility for any other derivatives. The microrotation is then,

$$
\phi_{z}(x)=\phi^{\prime}(x)+\phi_{z}^{\prime \prime}(x)=\frac{1}{2} \theta(x)-\frac{\gamma V(x)}{4 \varphi \kappa E_{m} I_{z}}
$$

The microcouple (moment per unit of volume exerted by the microstructure) is given by Equation (2),

$$
m_{z x}=\gamma \phi_{z, x}
$$

when Equation (70) is used,

$$
m_{z x}=\frac{\gamma}{2} \frac{\partial \theta(x)}{\partial x}
$$

That in turn produces Equation (54).

Equation (51) is now modified, to include $\varphi$,

a) $V_{a \rightarrow b}=V_{1}-F$

b) $M_{0 \rightarrow a}=V_{1} x-M_{1}$

c) $M_{a \rightarrow b}=V_{1} x-M_{1}-F(x-a)$

d) $\varphi E I \theta_{0 \rightarrow a}=\frac{V_{1} x^{2}}{2}-M_{1} x$

e) $\varphi E I \theta_{a \rightarrow b}=\frac{V_{1} x^{2}}{2}-M_{1} x-\frac{F(x-a)^{2}}{2}$

f) $\varphi E I v_{0 \rightarrow a}=\frac{V_{1} x^{3}}{6}-\frac{M_{1} x^{2}}{2}$

g) $\varphi E I v_{a \rightarrow b}=\frac{V_{1} x^{3}}{6}-\frac{M_{1} x^{2}}{2}-\frac{F(x-a)^{3}}{6}$

$$
F\left\{\begin{array}{c}
\frac{(L-a)^{2}}{2} \\
\frac{(L-a)^{3}}{6}
\end{array}\right\}=\left[\begin{array}{cc}
\frac{L^{2}}{2} & -L \\
\frac{L^{3}}{6} & -\frac{L^{2}}{2}-
\end{array}\right]\left\{\begin{array}{c}
V_{1} \\
M_{1}
\end{array}\right\}
$$

These are the equations that solve the case of the hyperstatic micropolar beam. The last two Equations (71) can now be assembled into a matrix equation, which when inverted,

$$
\left\{\begin{array}{l}
V_{1} \\
M_{1}
\end{array}\right\}=\left[\begin{array}{cc}
\frac{6}{L^{2}} & -\frac{12}{L^{3}} \\
\frac{2}{L} & -\frac{6}{L^{2}}
\end{array}\right] F\left\{\begin{array}{l}
\frac{(L-a)^{2}}{2} \\
\frac{(L-a)^{3}}{6}
\end{array}\right\}
$$

gives the usual response to the classic elasticity theory. Observe that $\varphi$ is not involved.

$$
\begin{gathered}
V_{1}=\left(1-\frac{a}{L}\right)^{2}\left[3-2\left(1-\frac{a}{L}\right)\right] F \\
M_{1}=\left(1-\frac{a}{L}\right)^{2}\left(\frac{a}{L}\right) F L
\end{gathered}
$$

If $a$ is the half of the beam,

$$
V_{1}=\frac{F}{2} \quad M_{1}=\frac{F L}{8}
$$


The maximum vertical displacement is found when $\theta(x)$ $=0$, from Equation (71),

$$
\begin{gathered}
0=\frac{V_{1} x^{2}}{2}-M_{1} x-\frac{F(x-a)^{2}}{2} \\
0=\left(\frac{V_{1}-F}{2}\right) x^{2}-\left(M_{1}-F a\right) x-F \frac{a^{2}}{2} \\
x=\frac{\left(M_{1}-F a\right)}{V_{1}-F} \pm \sqrt{\frac{\left(M_{1}-F a\right)^{2}}{\left(V_{1}-F\right)^{2}}+\frac{F a^{2}}{V_{1}-F}}
\end{gathered}
$$

That gives $x=L / 2$ when $a=L / 2$. The micropolar effect does not alter the localization of the maximum deformation. It affects only the value of the maximum displacement. For the case $a=L / 2$ one has,

$$
v_{\max }=-\frac{F L^{3}}{192 \varphi E_{m} I_{z}}
$$

\section{Conclusions}

The objective of this paper is to present a simple way to solve the micropolar isotropic material strength for engineering applications. The proposed solution, which is related to the response of this kind of medium to external loads, can be obtained by an iterative process that starts from a deformation field similar to the classic elasticity theory.

The two cases which were presented here demonstrate that this iterative process leads to a consistent solution of the constitutive micropolar isotropic equations; however, some experimental work is required to verify the results obtained.

In the clamped beam with a free end and a microcouple per unit of volume case, the mathematical solution is a strain field which is not observed in the classic elasticity theory; this strain field is a particular case of flexion where only shear strains exist. This occurs because the beam cross sectional planes do not rotate but slide over one another. The displacement causes macrorotations to equilibrate the microrotations which are in opposition to the distributed microcople per unit of volume.

The solution of the double clamped beam with a concentrated force case represents a double clamped beam with a concentrated force. It can be considered an extention of the Euler-Bernoulli beam to the micropolar elasticity. The beam shows to be stiffer than this clasic elasticity counterpart. This is because of the oppositon of the microrotations to the bending moment at each transversal section.

Departing from a solution well known in the equivalent case in the classical elasticity, it is quite easier to obtain the solution to the response of the micropolar medium. In addition, the physical interpretation is much simpler.

This approach may open the possibility of a strengthening of material science for microcontinuum media, through an iterative process to start solving classic, then micropolar elasticity, then solve microstretch cases and finally obtain the micromorphic elasticity solutions. Future articles are focused on experimented data on one hand and on the other hand to expand this kind of analysis to microstrech elasticity

\section{REFERENCES}

[1] R. Lakes, "Experimental Methods for Study of Cosserat Elastic Solids and Other Generalized Elastic Continua," In: H. Muhlhaus, Ed., Continuum Models for Materials with Micro-Structure, Wiley, New York, 1995, pp. 1-22.

[2] J. A. Beltran-Fernández, L. H. Hernández-Gómez, G. Urriolagoitia-Calderón, A. González-Rebatú and G. Urriolagoitia-Sosa, "Biomechanics and Numerical Evaluation of Cervical Porcine Models Considering Compressive Loads Using 2-D Classic Computer Tomography CT, 3-D Scanner and 3-D Computed Tomography,” Applied Mechanics and Materials, Vol. 24-25, 2010, pp. 287-295. doi:10.4028/www.scientific.net/AMM.24-25.287

[3] C. Eringen, "Polar and Nonlocal Field Theories," Continuum Physics, Academic Press, London, New York, 1976.

[4] R. Lakes, "Elastic and Viscoelastic Behaviour of Chiral Materials,” International Journal of Mechanical Sciences, Vol. 43, No. 7, 2001, pp. 1579-1589.

[5] J. Rosenberg, R. Cimrman and V. Novacek, "Bone Tissue Modelling Based on the Orthotropic Micropolar Continuum,” Engineering Mechanics, Svratka, 2002.

[6] C. Eringen, "A Unified Continuum Theory of Elctrodynamics of Liquid Crystals," International Journal of Engineering Science, Vol. 35, No. 12-13, 1997, pp. $1137-$ 1157. doi:10.1016/S0020-7225(97)00012-8

[7] C. Eringen, "A Unified Continuum Theory for Electrodynamics of Polymeric Liquid Crystals," International Journal of Engineering Science, Vol. 38, No. 9-10, 2000, pp. 959-987. doi:10.1016/S0020-7225(99)00089-0

[8] S. M. Clarke, A. R. Tajbakhsh, E. M. Terentjev, C. Remillant, G. R. Tomlinson and J. R. House, "Soft Elasticity and Mechanical Damping in Liquid Crystalline Elastomers”, Journal of Applied Physics, Vol. 89, No. 11, 2001, pp. 6530-6535. doi:10.1063/1.1368177

[9] R. D. Gauthier and W. E. Jahsman, "Bending of a Curved Bar of Micropolar Elastic Material,” Journal of Applied Mechanics, Vol. 42, No. 2, 1975, pp. 369-374. doi:10.1115/1.3423583

[10] A. C. Eringen, "Fracture, and Advanced Treatise," Academic press, New York, 1968.

[11] S. Ramezani, R. Naghdabadi and S. Sohrabpour, "Analysis of Micropolar Elastic Beams," European Journal of Mechanics-A/Solids, Vol. 28, No. 2, 2009, pp. 202-208. doi:10.1016/j.euromechsol.2008.06.006 


\section{Symbology}

$M=$ bending moment

$V=$ shear force

$x_{i}=$ coordinates in the $i$ direction

$E_{m}=$ micropolar Young modulus

$\sigma_{o}=$ proportional constant

$\phi_{t}=$ microrotation

$\theta=$ bending angle

$I_{i}=$ second moment of inertia around $i$

$\delta_{i j}=$ Dirac Delta

$t_{R L}=$ stress $\lambda, \mu, \kappa, \alpha, \beta \rho=$ constitutive parameter

$e_{r l}=$ symmetric strain tensor

$\varepsilon_{r l}=$ symmetric strain tensor

$m_{r l}=$ micromoments pro unit of area

$u_{i}=$ displacement in $i$ direction

$\rho=$ density

$f_{k}=$ force per unit of mass

$n_{i}=$ unitary vector in the $i$ direction

$R_{i}=$ macrorotation

$l_{k}=$ microcouple per unit of mass

$j=$ microinertia 